\title{
Three-dimensional super-resolution with a 4Pi-confocal microscope using image restoration
}

\author{
M. Schrader and S. W. Hell ${ }^{\text {a) }}$ \\ High Resolution Optical Microscopy Group, Max-Planck-Institute for Biophysical Chemistry, \\ D-37070 Göttingen, Germany \\ H. T. M. van der Voort \\ Scientific Volume Imaging B.V., J. Geradtsweg 181, 1222 PS Hilversum, The Netherlands
}

(Received 23 June 1997; accepted for publication 13 July 1998)

\begin{abstract}
The combination of two-photon excitation 4Pi-confocal fluorescence microscopy with image restoration leads to a fundamental improvement of three-dimensional resolution in the imaging of transparent, fluorescent specimens. The improvement is exemplified by randomly dispersed fluorescent beads and with actin filaments in a mouse fibroblast cell. For an illumination wavelength of $810 \mathrm{~nm}$, we obtained lateral and axial full-width at half-maxima of point-like objects of 120-140 $\mathrm{nm}$, and 70-100 nm, respectively. Fluorescent beads that are $150 \mathrm{~nm}$ apart are imaged with an intensity dip of $\sim 25 \%$. This amounts to a $\sim$ sixfold improvement of the axial resolution over standard two-photon confocal microscopy. In the cell, the 3D-images reveal details otherwise not resolvable with focused light. (c) 1998 American Institute of Physics. [S0021-8979(98)00920-7]
\end{abstract}

\section{INTRODUCTION}

Abbe's work on image formation revealed the limiting role of diffraction on the resolving power of a focusing light microscope. ${ }^{1}$ His insight initiated radically different approaches such as electron and scanning tip microscopy which have either abandoned the use of light or the idea of focusing.

The principle of the scanning tip has also been successfully applied to light microscopy where it is referred to as near-field scanning optical microscopy. ${ }^{2,3}$ Near-field optical microscopy overcomes the diffraction by confining the specimen-light interaction to a tiny area that is defined solely by the dimensions of the tip. This principle is applicable if the tip is placed at subwavelength proximity to the specimen. The resolution reported for these systems ranges from $150 \mathrm{~nm}$ down to remarkable values of a few nanometers. ${ }^{4-6}$

In spite of the outstanding merits of electron and scanning tip microscopes, they must be regarded as only a partial remedy for limited optical resolution. Electron microscopy for example, requires vacuum and an elaborate specimen preparation. ${ }^{7}$ Scanning tip imaging also depends on the performance of the tips so that tip manufacture is a major concern in this field of research.

While scanning tip methods have proven invaluable for surface physics and chemistry, one notices that they have been scarcely applied to biological imaging. This is largely due to the surface interaction. Investigations of thicker specimens, such as human cells, inevitably require a destructive sample preparation. In biological microscopy, however, it is usually more important to image the interior of cells, or even live cells, rather than cell surfaces. So far, this has been

a) Author to whom correspondence should be addressed; electronic mail: shell@gwdg.de satisfactorily achieved only with far-field light microscopy. The versatility of far-field light microscopy has also been supported by continuous progress in dye photochemistry and photophysics which has made it possible to label proteins, DNA, and other cell organelles with nontoxic fluorescent markers. Highly specific fluorescence imaging of the structure and function of fixed and live cells has become a standard method in molecular biology. In fact, about $80 \%$ of all imaging applications in biology are based on fluorescence. ${ }^{8}$ It can be seen that electron and scanning tip microscopes have not replaced the light microscope as the most popular tool in the biological sciences.

The continuing popularity of the light microscope has triggered a series of inventions, the most successful of which is the scanning confocal microscope. ${ }^{9-11}$ In contrast to the regular light microscope, the confocal microscope employs a point-like illumination and detection arrangement. Therefore the point-spread function (PSF) of a confocal fluorescence microscope is determined by the product of two almost identical intensity PSFs, namely the focal 3D-intensity diffraction pattern of the illumination light and a similar one accounting for imaging into the detection pinhole. ${ }^{10}$ For circularly polarized illumination, both differ only by the wavelength accounting for the Stokes shift of the dye. Hence, as a good approximation the effective PSF of a confocal microscopes is given by the square of the focal illumination intensity distribution in the objective lens so that, in a simplistic photon picture, only photons from the closest vicinity of the diffraction limited spot contribute to the signal. In a confocal microscope, the effective focus acts as a threedimensional probe which can be scanned through a transparent specimen. Therefore, confocal fluorescence microscopy readily produces (3D-) images of transparent specimens such as cells. 
A quadratic effective PSF can also be achieved by exciting the dye through nonresonant two-photon absorption. ${ }^{12,13}$ In this case, one is able to obtain similar 3D-imaging capabilities using only excitation. An important benefit of twophoton excitation microscopy is the accessibility of UV fluorophores without requiring UV-optical components. The viability of multiphoton excitation microscopy is catalyzed by the high local intensities achieved in a high aperture microscope. Recently, even three-photon excitation microscopy has been demonstrated ${ }^{14}$ and applied to the imaging of biological specimens and even live cells. ${ }^{15,16}$ When considering the effect of multiphoton excitation on the resolution one has to bear in mind that two- or three-photon excitation usually calls for a doubled or tripled excitation wavelength resulting in a spatially enlarged focus. The nonlinearity of the excitation is not able to fully compensate for this effect, so that practical multiphoton images are generally poorer in resolution than their confocal single-photon counterparts. ${ }^{17}$

The advantages of focusing over near-field optics provided a great incentive for us to readdress the resolution issue in the far field. ${ }^{18,19}$ The advent of 3D imaging through confocal and two-photon excitation microscopy in the eighties and early nineties, highlighted the obvious fact that in a transparent three-dimensional object, the resolution is a three-dimensional problem. Even for the highest numerical apertures, the resolution along the optic axis is about 3 to 4 times poorer than its focal plane counterpart. ${ }^{20}$ In standard confocal and multiphoton microscopes, typical resolution is of the order of $150-300 \mathrm{~nm}$ in lateral and of $500-1000 \mathrm{~nm}$ in axial direction. ${ }^{21}$

An approach that allows increased axial resolution is standing-wave microscopy. This uses flat counterpropagating coherent beams to produce a flat standing wave pattern in the focal region of the lens. This is achieved technically either by placing a mirror in the focal region of the lens from which the wave fronts bounce back into the focal region, or by using two objective lenses with flat-wave illumination. ${ }^{22}$ The unfocused wave fronts produce a periodic pattern of flat standing waves in the excitation field that is useful for discriminating objects closer than $\lambda / 2$. However, they do not allow for 3D imaging since they require the objects to be thin of the order of $\lambda / 2$ to avoid ambiguities in the periodic standing wave pattern. Another endeavor for increasing axial resolution is confocal theta microscopy. ${ }^{23,24}$ This system uses two orthogonal low aperture lenses and is able to provide approximate isotropic 3D resolution. The drawback of this approach is that the resulting focal 3D volume is generally larger than that of a high aperture lens, as the lateral resolution is poorer than in a high aperture confocal arrangement.

In previous articles, we pointed out the potential of combining image restoration and confocal microscopy for increasing the resolution in far-field light microscopy. ${ }^{25}$ In this article, we follow the approach of synergistically combing two-photon excitation 4Pi-confocal microscopy ${ }^{18,19,26}$ with image restoration. ${ }^{27-29} \mathrm{We}$ provide further evidence that this method is capable of achieving three-dimensional resolution of the order of $100 \mathrm{~nm}$. We shall begin with a review of the concept of 4Pi-confocal microscopy and the restoration approaches. In Sec. IV we shall display 3D images of test ob-

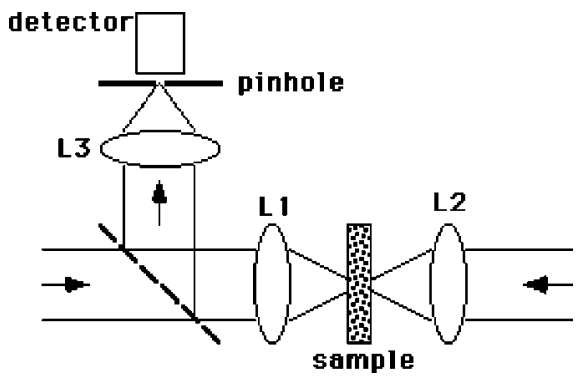

FIG. 1. Scheme of the 4Pi-confocal microscope of type A.

jects as well as of parts of the filamentous actin in the cell cytoskeleton of a mouse fibroblast cell, and compare the resolution and imaging performance of two-photon confocal, $4 \mathrm{Pi}$-confocal and restored 4Pi-confocal microscopes.

\section{THE CONCEPT OF 4Pi-CONFOCAL MICROSCOPY}

The primary goal of 4Pi-confocal microscopy is the increase of far-field resolution in the axial direction. For this purpose, the 4Pi-confocal microscope uses two opposing objective lenses of high numerical aperture with which the same object point is coherently illuminated and/or observed. ${ }^{18,19}$ The two spherical wave fronts add up to a single wave front so that the total aperture angle is doubled. This is accomplished either for illumination, or detection, or simultaneously for both. With two objective lenses it is not possible to obtain a complete solid angle of $4 \pi$. The acronym $4 \mathrm{Pi}$, however, is a reminder of the basic idea behind the concept.

Figure 1 sketches the 4Pi-confocal microscope for the case of coherent illumination through both objective lenses; the collection is accomplished through a single lens. This imaging mode is technically the simplest version of $4 \mathrm{Pi}$ confocal microscopy and is referred to as $4 \mathrm{Pi}$-confocal microscopy of type A. The light source is, preferably, a laser whose beam is split into two coherent partial beams. The focal intensity distribution of a 4Pi-illumination PSF is calculated by the coherent summation of the two focal electric fields of the two opposing lenses. We begin with the calculation of the 4Pi-confocal PSF. Following Richards and Wolf, ${ }^{30}$ the electric field in the focal region of a lens is given by

$$
\begin{aligned}
\mathbf{E}(u, \nu, \varphi) & =\left(e_{x}, e_{y}, e_{z}\right) \\
& =-i\left[I_{0}+I_{2} \cos (2 \varphi), I_{2} \sin (2 \varphi),-2 i I_{1} \cos \varphi\right],
\end{aligned}
$$

with $I_{0,1,2}=I_{0,1,2}(u, v)$ being integrals over the lens aperture defined in Ref. 30 . The integrals $I_{1,2}$ consider the high angle effects as required when precisely calculating the focused field of a high aperture system. The parameters $u$ and $v$ are optical units defined as $u=(2 \pi / \lambda) n z \sin ^{2} \alpha$, and $v$ $=(2 \pi / \lambda) n r \sin \alpha$. The angle $\varphi$ is between the direction of vibration of the incident electric field and the direction of observation. The angle $\alpha$ denotes the semiaperture angle and 
$\lambda$ is the wavelength. $J_{0,1,2}$ are Bessel functions of the first kind. The focal intensity for a single lens is given by the modulus square of the electric field,

$$
\begin{aligned}
h(u, \nu, \varphi)= & |\mathbf{E}(u, \nu, \varphi)|^{2} \\
= & \left|I_{0}\right|^{2}+\left|I_{2}\right|^{2}+4\left|I_{1}\right|^{2} \cos ^{2} \varphi \\
& +2 \operatorname{Re}\left\{I_{0} I_{2}^{*}\right\} \cos 2 \varphi
\end{aligned}
$$

with Re denoting the real part. For randomly or circularly polarized light, the dependence on $\varphi$ vanishes and the normalized PSF is reduced to

$$
h(u, \nu)=\left|I_{0}\right|^{2}+2\left|I_{1}\right|^{2}+\left|I_{2}\right|^{2} .
$$

For low apertures, Eq. (3) becomes $h(u=0, v) \cong\left|I_{0}\right|^{2}$ which is the Airy intensity distribution that is commonly used to describe the classical resolution limit in the focal plane. ${ }^{30}$ The 4Pi-illumination PSF is readily calculated by adding the electric fields. ${ }^{19}$ Constructive interference in the geometric focus yields

$$
h^{4 \mathrm{Pi}}(u, \nu, \varphi)=\left|\mathbf{E}_{1}(u, \nu, \varphi)+\mathbf{E}_{2}(-u, \nu, \varphi)\right|^{2},
$$

where $\mathbf{E}_{1}$ and $\mathbf{E}_{2}$ are the fields from the two lenses. Further evaluation results in

$$
\begin{aligned}
h^{4 \mathrm{Pi}}(u, \nu, \varphi)= & \operatorname{const}\left(\operatorname{Re}\left\{I_{0}\right\}^{2}+\operatorname{Re}\left\{I_{2}\right\}^{2}\right. \\
& +2 \operatorname{Re}\left\{I_{0}\right\} \operatorname{Re}\left\{I_{2}\right\} \cos 2 \varphi \\
& \left.+4 \operatorname{Re}\left\{I_{1}\right\}^{2} \cos ^{2} \varphi\right) .
\end{aligned}
$$

For random polarization, we obtain

$$
h^{4 \mathrm{Pi}}(u, \nu)=\operatorname{const}\left(\operatorname{Re}\left\{I_{0}\right\}^{2}+2 \operatorname{Re}\left\{I_{1}\right\}^{2}+\operatorname{Re}\left\{I_{2}\right\}^{2}\right) .
$$

Equation (6) is the 4Pi-illumination PSF describing the intensity of the excitation light. It is the counterpart of Eq. (3) in the single lens case. The numerical computation of $h^{4 \mathrm{Pi}}(u, v)$ renders a main maximum and axial lobes, ${ }^{19}$ the height and number of which are a function of the semiaperture angle $\alpha_{\max }$. The height of the lobes decreases with increasing $\alpha_{\max }$; for a complete spherical wavefront of $4 \pi$, the lobes vanish. It is desirable to have the highest possible aperture however this is limited by manufacturing constraints. At present the largest aperture possible is obtained using oil immersion lenses having a semiaperture angle of $\alpha_{\max }$ $\cong 3 / 8 \pi$. For constructively interfering wavefronts and $\alpha_{\max }$ $\cong 3 / 8 \pi$, the $4 \mathrm{Pi}$-confocal PSF results in a main maximum and an axial lobe of $\sim 60 \%$ above and below the focal plane. ${ }^{19}$ The main maximum of the 4Pi-illumination PSF [Eq. (6)] is about four times narrower than its single lens counterpart [Eq. (3)], thus promising a fundamentally increased axial resolution. The lobes compromise the benefit of the narrow main maximum and need to be suppressed to avoid ambiguous imaging along the optic axis.

Figure 2 shows a comparison of the (a) confocal PSF with the (b) 4Pi-confocal PSF as surface intensity plots along the optic axis. The PSFs have been measured by scanning a scattering gold particle of $50 \mathrm{~nm}$ diameter through the focus of the microscopes. ${ }^{31}$ We used a wavelength of $633 \mathrm{~nm}$ as emitted by a HeNe laser. The numerical aperture of the

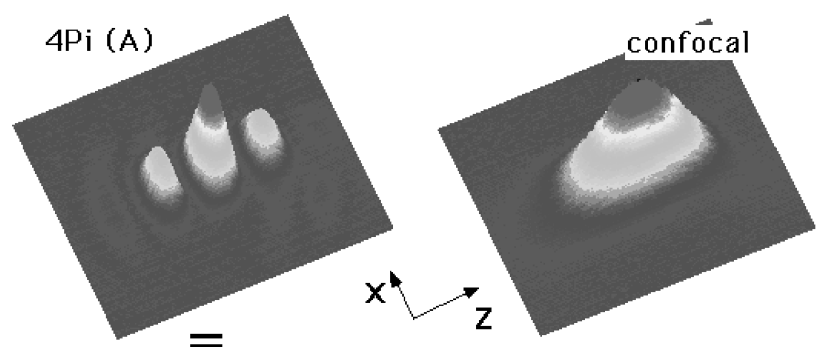

(a)

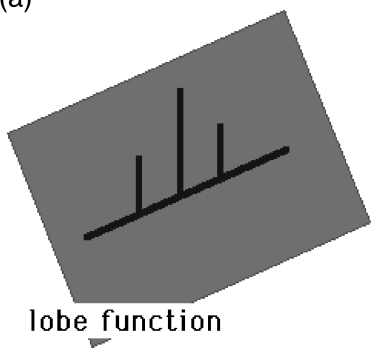

(c) (b)

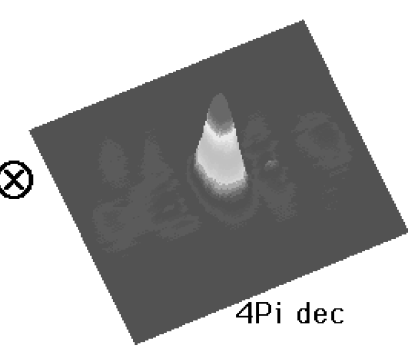

(d)

FIG. 2. Intensity surface plots of the PSF of the (a) 4Pi-confocal, (b) confocal, and the (d) point-deconvolved $4 \mathrm{Pi}$-confocal microscope as determined with light scattering beads. The plot in (c) represents the lobe function, $I(z)$. The arrangement of the figures symbolizes the fact that the $4 \mathrm{Pi}-$ PSF can be approximated by the convolution of the peak function of (d) with the lobe function of (c). The comparison between (b) and (d) reveals a 4.5-fold improvement of the axial resolution in 4Pi-confocal microscopy over regular confocal microscopy.

lenses was 1.4 oil immersion. In the 4Pi-confocal case, one can clearly recognize the pronounced main maximum and the axial lobes.

A further increase of the semiaperture angle is desirable but is hampered by technical challenges in lens manufacturing. Hence, the problem of the lobes must be addressed differently. A useful approach is the multiphoton excitation of the dye in conjunction with confocal detection through a pinhole. Multiphoton excitation reduces the contribution of the secondary maxima because of the higher order dependence of the excitation on the illumination intensity. The use of a confocal pinhole suppresses the lobes as it reduces the probability of out-of-focus fluorescence photons being detected and therefore also contributions from axial lobes. For confocal detection through a single lens (Fig. 1) the detection process is described by a single lens PSF [Eq. (3)] calculated for the fluorescence wavelength. Thus, the point-spread function of a two-photon confocal fluorescence 4Pi-confocal microscope is given by

$$
h_{\text {conf }}^{4 \mathrm{Pi}}(u, \nu)=\left|h_{\text {ill }}^{4 \mathrm{Pi}}(u / 2, \nu / 2)\right|^{2} h_{\mathrm{det}}(u, \nu) .
$$

In fact, the combination of two-photon excitation and confocal detection is particularly advantageous ${ }^{32}$ as the double excitation wavelength also doubles the distance between the maximum and the axial lobes. As a result, the lobes are at twice the distance from the focal plane so that the confocal detection efficiently reduces the height of the lobes. In theory, one could suppress the lobes well below $10 \%$. In practice, however, for a two-photon excitation wavelength at about $800 \mathrm{~nm}$ and a medium detection wave- 

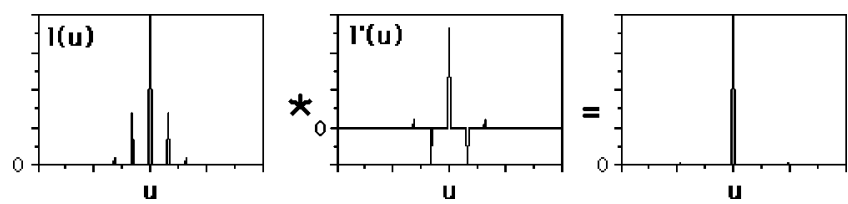

FIG. 3. Point deconvolution for axial lobe removal.

length of about $580 \mathrm{~nm}$, the refractive index mismatch between the sample and the immersion medium render lobes in the range of $25 \%-40 \% .^{33,34}$

The incompleteness of lobe suppression is not necessarily a major obstacle for 4Pi imaging, since the lobes can also be removed computationally. Provided they are well below $50 \%$ one can apply a fast deconvolution algorithm. ${ }^{34}$ This can be understood on the basis that, as a good approximation, the axial profile of the 4Pi-confocal PSF is a convolution of two functions: the peak intensity function, $\left|h_{\text {peak }}(u, v)\right|^{2}$ describing a single interference maximum [Fig. 2(d)], and the lobe function, $l(u)$ representing the location and the height of the (three) peaks [Fig. 2(c)]. The approximation holds as long as the relative height of the lobes does not depend on the lateral position within the PSF. In case of a two-photon 4Pi-confocal PSF with excitation at $810 \mathrm{~nm}$ and detection at $580 \mathrm{~nm}$ which is close to our experimental conditions, the relative height of the lobes on the optical axis is $19.9 \%$. At a lateral position where the PSF at $z=0$ has dropped to $50 \%$, the lobes are at about $21.9 \%$ and they reach $29.6 \%$ at a lateral position where the PSF at $z=0$ has dropped to $10 \%$. The absolute error introduced by this deviation from the approximated shape is about $1 \%$.

The lobe function is actually the sum of weighted $\delta$ distributions representing the main maximum and the lobes. The inverse function $l^{\prime}(u)$ of the lobe function can be readily expressed as $3-5$ weighted $\delta$ distributions with changing sign (Fig. 3). In theory, the inverse filter consists of more peaks but for lobes smaller than $30 \%$ they are negligible with respect to the noise that is usually present in a real image. This simple inverse filter allows for a rapid on-line deconvolution. ${ }^{34}$ After the point deconvolution, the effective PSF of the 4Pi microscope is a single peak that is about four times narrower than that of a single lens high aperture microscope [Figs. 2(d) and 3]. The narrower main maximum of the 4Pi-confocal microscope accounts for an axial resolution improvement by a factor of four in far-field fluorescence microscopy.

\section{RESOLUTION IMPROVEMENT THROUGH IMAGE RESTORATION}

In fluorescence imaging, the phase of the excitation light is lost upon absorption, so that the image $g\left(\mathbf{r}^{\prime}\right)$ is given by the three-dimensional convolution ${ }^{20,27}$ of the PSF, $h(\mathbf{r})$, with the unknown object function, $f(\mathbf{r})$. The latter represents the fluorochrome distribution of the specimen in the object space:

$$
g\left(\mathbf{r}^{\prime}\right)=h(\mathbf{r}) \otimes f(\mathbf{r}) .
$$

Here, we express the PSF [Eqs. (3) and (6)] in spatial coordinates $\mathbf{r}=(x, y, z)$ and as a scalar function that is ideally translation invariant in the object volume. With a noise-free image and a perfectly known PSF one could, in principle, use direct deconvolution, i.e., inverse filtering, to recover the distribution of the fluorophore in the object with a spatial frequency content up to the bandlimit of the microscope.

Unfortunately, this approach is hardly applicable. The limited signal to noise ratio (SNR), the limited knowledge of the real PSF of the microscope and the bandlimited character of the PSF renders a direct deconvolution unfeasible. Still, it is possible to significantly improve the resolution by nonlinear deconvolution algorithms. ${ }^{28,35}$ In fact, these algorithms do not deconvolve the image directly but in an iterative manner. During iterations the match between the measured image and a synthetic image of the current object estimate is step by step improved. The synthetic image is computed by convolving the object estimate with a previously measured PSF. Such iterative nonlinear restoration techniques allow reconstruction of spatial frequencies which were lost in the imaging process, e.g., the "missing cone"' spatial frequencies of a wide field microscope. We emphasize here that due to the nonlinear character the efficiency of spatial frequency reconstruction is highly object dependent.

In spite of being well-established in microscopy, restoration algorithms have only recently succeeded in significantly improving the resolution. ${ }^{28,36}$ This is due to augmented computational speed of current computers which now enables the application of stable algorithms to meaningful 3D data sets. In particular, the combination of high sensitivity detection with piezoelectric stage scanning microscopy has made possible an increase of resolution by 1.5 and 3 in lateral and axial direction, ${ }^{37}$ respectively, as demonstrated by imaging $F$-actin fibers in mouse fibroblast cells.

When applying restoration techniques one has to take the effect of noise into account. At signal levels of about 0-200 counted photons per pixel, the noise generally obeys a Poisson distribution. The presence of noise can be modeled as

$$
m\left(\mathbf{r}^{\prime}\right)=N\left[g\left(\mathbf{r}^{\prime}\right)\right],
$$

where $m\left(\mathbf{r}^{\prime}\right)$ is the recorded image. The function $N$ accounts for the noise in the signal. ${ }^{38}$ The restoration procedure computes a new estimate of the object function for each iterative step. The criterion used in matching the synthetic image of the object estimate with the recorded image largely determines the outcome of the restoration. The choice of this criterion, or the distance measure, is based on a priori known properties of the object function and the imaging. One of the a priori known properties is that the object function cannot be negative since it represents a distribution of fluorophores. For non-negative object functions, the $I$-divergence distance measure

$$
I(a \| b)=a \ln [a / b]-(a-b)
$$

is the only possible measure that is consistent with a limited set of plausible axioms. ${ }^{39}$ The functions $a$ and $b$ denote the functions to be compared. It has also been shown that minimizing the $I$ divergence is equivalent to maximizing the logarithmic likelihood, $l$, of the estimate $f^{\prime}$ given the measured image $m\left(\mathbf{r}^{\prime}\right)$ : 


$$
l\left(f^{\prime}\right)=-\int_{R^{\prime}} g^{\prime}\left(\mathbf{r}^{\prime}\right) d \mathbf{r}^{\prime}+\int_{R^{\prime}} \ln \left[g^{\prime}\left(\mathbf{r}^{\prime}\right)\right] m\left(\mathbf{r}^{\prime}\right) d \mathbf{r}^{\prime},
$$

$R^{\prime}$ represents the image space and $g^{\prime}\left(\mathbf{r}^{\prime}\right)$ the imaged estimate. ${ }^{40}$ The function $f^{\prime}$ which maximizes the logarithmic likelihood is found with the expectation-maximization (EM) algorithm.

$$
f^{\prime k+1}(\mathbf{r})=f^{\prime k}(x) \int_{\mathbf{r}^{\prime}}\left[\frac{h\left(\mathbf{r}-\mathbf{r}^{\prime}\right)}{\int_{\mathbf{r}} h\left(\mathbf{r}^{\prime}-\mathbf{r}\right) f^{\prime k}(\mathbf{r})+B}\right] m\left(\mathbf{r}^{\prime}\right) d \mathbf{r}^{\prime}
$$

in which $B$ is the average of a homogeneous background. The solution found by the EM-maximum likelihood estimation (MLE) algorithm is regularized through the use of the method of Gaussian sieves. ${ }^{41}$ In the results shown, a sieve with a sigma of 0.2 sample interval was applied after each fifth iteration of the EM-MLE algorithm. This sieve effectively prevents construction of out-of-band spatial frequency components. Subsequently, the $I$ divergence of the imaged estimate and the recorded image was computed and compared to the previous value. The iterations were stopped when the relative change of the $I$ divergence dropped below $0.01 \%$. The EM-MLE algorithm was chosen since it was found that for low light levels the EM-MLE method offers superior performance over the widely used iterative constrained Tikhonov-Miller methods. ${ }^{38}$ The application of restoration algorithms in 4Pi-confocal microscopy is attractive, as the 4Pi-confocal microscope offers a four fold improved axial bandwidth in the optical transfer function of the microscope. ${ }^{42}$

\section{RESULTS}

We built a 4Pi-confocal microscope of type A employing a mode-locked Ti:sapphire laser (Mira 900F, Coherent Inc.) in order to enable efficient two-photon excitation (Fig. 1). The laser emits $150 \mathrm{fs}$ pulses at a repetition rate of 76 MHz. The pulses are spread to about $250 \mathrm{fs}$ in the focus of the objective lens as a result of the dispersion in the microscope. ${ }^{43}$ Nevertheless, efficient two-photon excitation of rhodamine and rhodamine derivatives can be performed at a wavelength ranging from 750 to $860 \mathrm{~nm}$. The fluorescence wavelength is largely between 550 and $630 \mathrm{~nm}$ and was separated through the dichroic beamsplitter and $2 \mathrm{~mm}$ of blue glass (Schott, BG39). The latter suppresses the laser light by about 10 orders of magnitude which was sufficient to completely suppress the contribution of the laser light to the background. Our experiments were performed with standard objective lenses (Leica 100x, Planapochromat); the lenses were not preselected by the manufacturer. The $4 \mathrm{Pi}-$ confocal microscope can be transformed to a regular confocal microscope by blocking one of the lenses.

First, we carried out experiments with fluorescence latex beads (Molecular Probes, Eugene, Oregon). The beads were $110 \mathrm{~nm}$ in diameter and were mounted in Aquatex (Merck, Darmstadt), which after a drying period of 3 to 4 weeks, has an index of refraction close to that of oil or glass (1.5). The excitation wavelength was $760 \mathrm{~nm}$. The average laser intensity was typically 1 to $2 \mathrm{~mW}$. The detection pinhole was 10

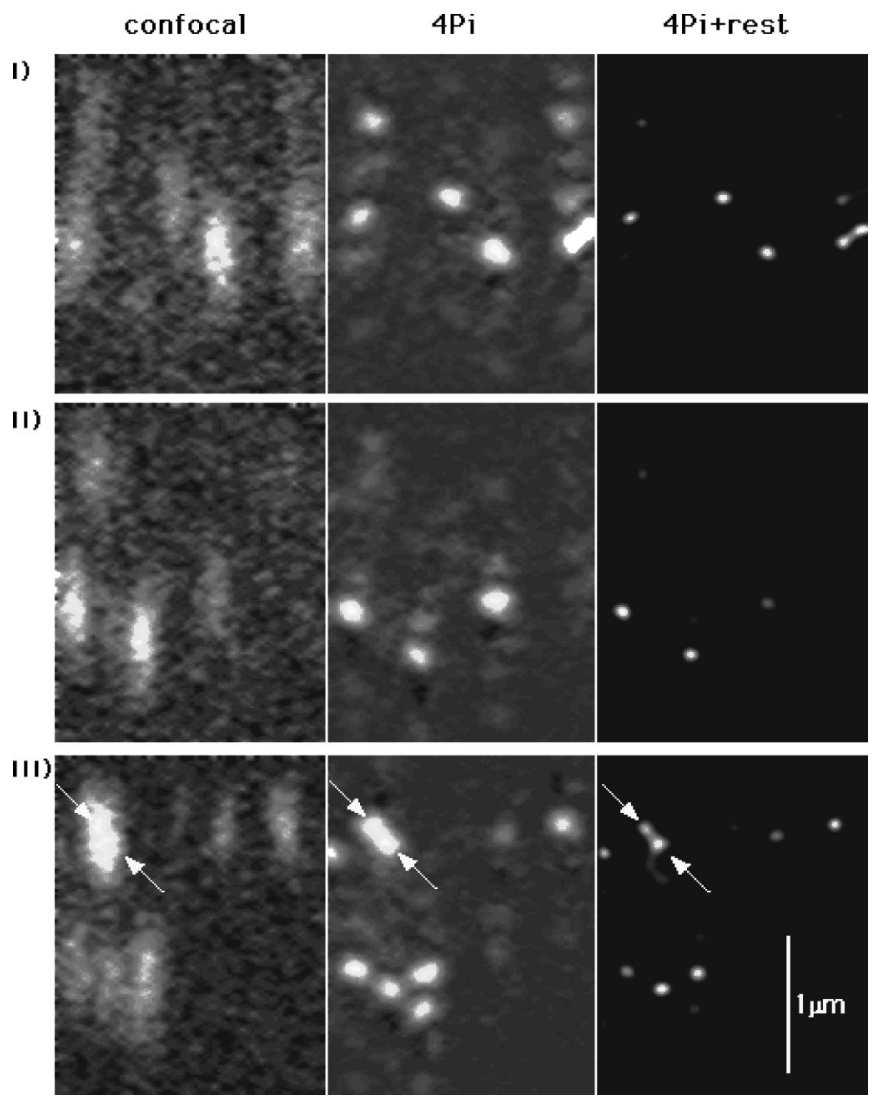

FIG. 4. Comparison of (two-photon) confocal, 4Pi-confocal, and 4Piconfocal restored axial images of randomly dispersed subresolution fluorescence beads. To allow a direct comparison, the same site of the specimen is imaged. For each imaging mode, three $X Z$ layers are shown (top to bottom). The layers are separated by $180 \mathrm{~nm}$ in the $Y$ direction. The comparison reveals a fundamental improvement of resolution in three-dimensional focusing light microscopy in the restored 4Pi-confocal case (see arrows).

$\mu \mathrm{m}$ in size, which in our case, amounted to about one quarter of the back projected Airy disk of the fluorescence light. Imaging was performed by scanning the sample with a 3Dpiezo stage featuring a scanning precision of $10 \mathrm{~nm}$ (Nanoblock, Melles Griot, Cambridge, UK). This precision was ensured by operating the stage in a closed loop. The first objective lens (L1) is fixed. The detection pinhole can be aligned with respect to the focus of the objective L1 by maximizing the two-photon fluorescence signal of a rhodamine sea. The focus of the second lens (L2) was adjusted with $10 \mathrm{~nm}$ precision piezoelectrically with respect to that of L1. The alignment can be carried out while observing the fluorescence signal of an axially scanned bead. Due to the interference the signal height is very sensitive to lateral and axial displacements of the second lens. At the same time the phase of the interference can be observed and adjusted.

We typically recorded 3D-data stacks. The stacks consisted of $X Z$ images, equidistant in the $Y$ direction. Figure 4 (left column) shows three single lens two-photon confocal axial images $(X Z)$ from a typical 3D-image stack. The pixel size was $36 \mathrm{~nm}$ in $X$ and $25 \mathrm{~nm}$ in $Z$. The data stack consisted of ten $X Z$ images. Three of them are shown in Fig. 4 to illustrate the $3 \mathrm{D}$ structure in the object. The distance between the layers was $180 \mathrm{~nm}$ ( $Y$ direction). In the two-photon confocal image, the beads were imaged as elongated patches. 
The elongation stems from the fact that the axial resolution is about 3 to 4 times poorer than the resolution in the focal plane. As the beads have a diameter of about $110 \mathrm{~nm}$ the bead image function is very close to the experimental PSF of the microscope. The arrows indicate a place in the sample where two beads are obviously not separated.

The center panel shows the corresponding three layers of the two-photon 4Pi-confocal 3D-data stack, after the point deconvolution. The 4Pi recording was performed at the same place in the sample with similar recording conditions, except for the doubled illumination power resulting from the simultaneous illumination through both lenses. This resulted also in an increased signal to noise ratio in the 4Pi case. The relative phase of the two interfering spherical wave fronts was adjusted prior to the imaging, by monitoring the axial response of a single isolated bead. From the single bead image we were also able to establish the width of the main maximum of the 4Pi-PSF and the height of the lobes. We found a full-width at half-maximum (FWHM) of $140 \mathrm{~nm}$ in axial direction and $180 \mathrm{~nm}$ in the focal plane. The relative height of the lobes was about $30 \%$ so that we could remove the lobes in the image by applying a fast point deconvolution.

When comparing the axial FWHM of the two-photon $4 \mathrm{Pi}$ confocal with that of the single lens confocal microscope, namely 140 to $640 \mathrm{~nm}$, one establishes a resolution improvement of 4.5. Along the marked line the beads are not separated by the confocal, whereas the $4 \mathrm{Pi}$-confocal image indicates that there is more than one bead. This can be seen more clearly in Fig. 5 where the data are shown as intensity profiles. The advantage of the point deconvolution is the high speed of the procedure. We applied the point deconvolution after the data acquisition although it could have been carried out on-line. The point deconvolution however, does not fully exploit the knowledge of the PSF, and, therefore does not provide the highest possible resolution. Hence, we adopted 3D-image restoration to obtain a further improvement in resolution.

Restoration of the image requires precise knowledge of the experimental 4Pi-confocal PSF. In principle, one could use the 3D image of an isolated bead for the PSF. The dimensions of the bead $(110 \mathrm{~nm})$ and the FWHM of the dye distribution object function of the bead are comparatively small with respect to the FWHM of the maxima of the 4PiPSF. Hence, the image of a single bead would be a good approximation for the experimental 4Pi-PSF. Even so, we decided to reduce the potential influence of the finite size of the beads.

We retrieved the 4Pi-PSF from the bead image by restoring the bead image with the object function of the beads. As a good approximation, the bead can be represented by a uniformly dyed sphere of $110 \mathrm{~nm}$ diameter. Therefore, we calculated the 4Pi-PSF by restoring the 3D-bead image with the known object function. Again, this is achieved by approaching the 4Pi-confocal bead image with a 4Pi-PSF gained by the MLE procedure with the condition that the bead object function is a uniformly dyed sphere.

The right hand panel of Fig. 4 shows the corresponding three layers of the 3D 4Pi-confocal data set after restoration

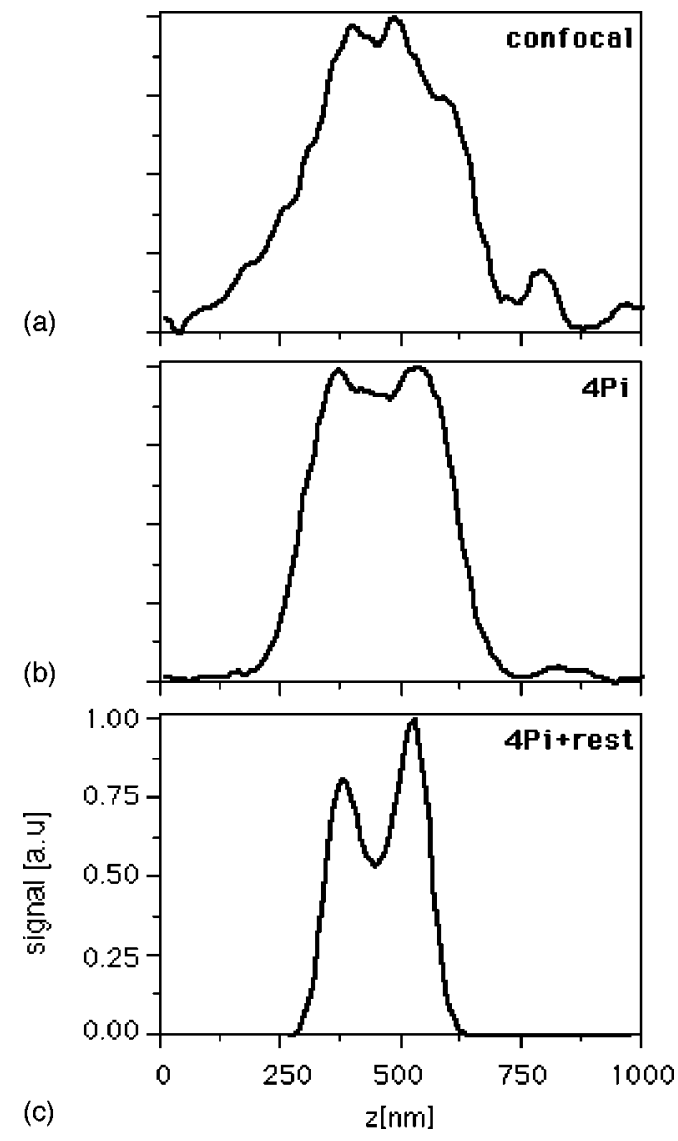

FIG. 5. Axial intensity profiles corresponding to the marked lines (arrows) of Fig. 4. The confocal and 4Pi-confocal lines are averaged across three pixels in lateral direction. The "dents" in the confocal profile are due to the poorer signal-to-noise ratio of the recording and subsequent averaging. The profiles demonstrate the improved resolution of 4Pi-confocal and restored $4 \mathrm{Pi}$-confocal microscopy. Note the intensity drop of $\sim 25 \%$ between beads that are $150 \mathrm{~nm}$ apart.

with the 4Pi-PSF. The full axial bandwidth of the 4Pi-PSF is exploited for the restoration of the images. This results in a strong increase in axial and lateral resolution as can be noticed from the comparison of the restored bead images. For instance in Fig. 4 (bottom, center and right), one can distinguish four beads that are separated by about $250 \mathrm{~nm}$ in lateral direction and axial direction. In the right hand panel, one of the beads is weaker because it is laterally out of focus. We evaluated restored single bead images in the whole data stack and found a FWHM of the bead images of $80 \mathrm{~nm}$ in the lateral and $70 \mathrm{~nm}$ in the axial direction. Taking into account the two-photon excitation wavelength of $760 \mathrm{~nm}$, this amounts to about one tenth of the wavelength. Thoughtfully applied, the FWHM can be a helpful criterion for the estimation of the resolution, however, the decisive criterion for the resolution is the ability to distinguish two adjacent objects.

The visual impression of fundamentally improved resolution is further substantiated by the intensity profiles, shown in Fig. 5. The intensity profiles of Fig. 5 reveal the data along the marked lines of Fig. 4 and impressively demonstrate the improvement in resolution obtained with two-photon 4Piconfocal and restored 4Pi-confocal microscopy over the standard two-photon confocal. As compared to the 4Pi-confocal image, the dip in the profile is about $25 \%$ deep and much 
more pronounced in the restored case [Fig. 5(c)]. The 110nm-diam beads are only $\sim 150 \mathrm{~nm}$ apart (center to center), but clearly distinguished. We calculated that in a confocal microscope, a similar dip of $25 \%$ would occur at a distance of $850 \mathrm{~nm}$.

The next step was to apply our method to biological specimens, in this case mouse skin fibroblast cells. The $F$ actin filaments of these cells were labeled with rhodaminephalloidin which has a two-photon absorption peak at around $810 \mathrm{~nm}$. We also used a confocal pinhole for detection with a diameter of $20 \mu \mathrm{m}$ amounting to about $50 \%$ of the size of the back projected Airy disk. The use of a confocal pinhole results in a loss in fluorescence intensity which we compensated for by applying a photon counting avalanche photodiode featuring detection quantum efficiencies in the order of $60 \%-70 \%$ at about $600 \mathrm{~nm}$ (EG\&G, SPCM-AQ-131). The average excitation power of the pulsed light was $1 \mathrm{~mW}$ which was not harmful to our sample. Typical pixel dwell time in our images was $2 \mathrm{~ms}$. The combination of high sensitivity detection with comparatively low excitation power reduced the potential effect of photobleaching.

Apart of being placed between two cover slips, the cell specimen was prepared according to published protocols for cell fixation. During the fixation the water was removed and replaced by glycerol. From the optics viewpoint there is now a significant difference between the medium refractive index of the sample. That of the sample is now close to glycerol ( $n=1.46)$ which is significantly less than that of the glass/ immersion system $(n=1.51)$. The difference in refractive index results in a slight spherical aberration and also influences the relative phase of the interfering spherical wavefronts. The spherical aberration leads to somewhat higher lobes, which are typically $40 \%-45 \%$ in the sample. The shift in the relative phase occurs when the specimen is moved along the optic axis. For instance, when the (optically thinner) sample is moved towards the lens L2, the optical path on the right is reduced, and vice versa.

We have found that the change of phase is a linear function of the axial movement which itself is a result of the refractive index step between the specimen and the cover glass/oil immersion medium. A complete turn of phase occurred for an axial translation of about $2.6 \mu \mathrm{m}$. The linearity of the phase shift can also be calculated by considering the refractive index step between the sample and the cover glass. The linearity of the phase change in the cytoskeleton is due to the fact that, after fixation, the cell consists mostly of glycerol (90\%-95\%). The cytoskeleton can thus be modeled by a uniform glycerol layer with small fluorescent objects. The linearity of the phase shift allowed us to compensate for the change in phase by applying a linear voltage ramp to a piezoelectrically driven mirror in one of the illumination paths.

Figure 6 shows $X Z$ images of actin fibers at the same site of the fibroblast cell. It is known that the diameter of the actin filaments and fiber bundles is about $7-30 \mathrm{~nm}^{7}$ Together with the label, the maximum diameter of the actin fibers can be calculated to be about $20-50 \mathrm{~nm}$. Figure 6(a) depicts the single lens confocal image while Fig. 6(b) shows the $4 \mathrm{Pi}$-confocal counterpart with subsequent restoration

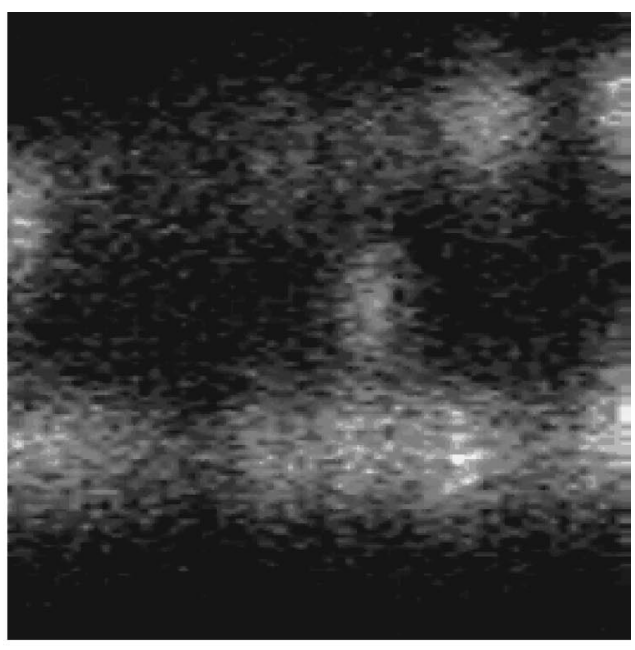

(a)

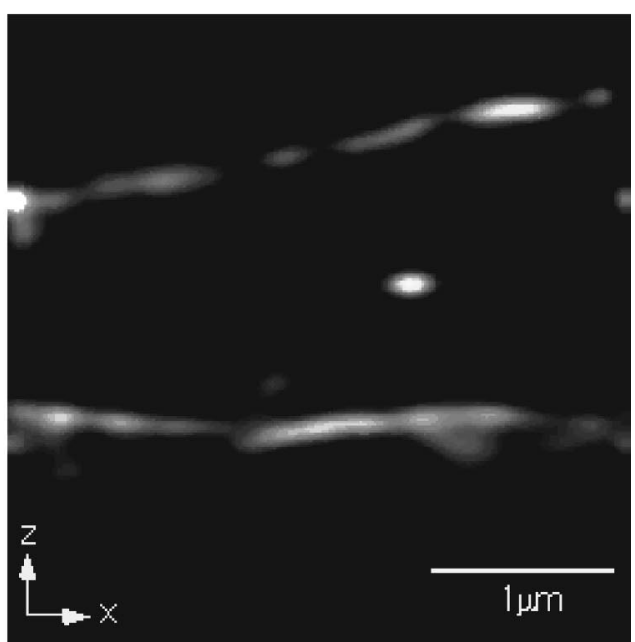

FIG. 6. Axial image through the $F$-actin filaments of a mouse skin fibroblast cell showing (a) the confocal and (b) the 4Pi-confocal restored case. Both $3 \mathrm{D}$ images were recorded at the same site of the specimen to allow a direct comparison of both methods. The $F$-actin fibers are directed along the $Y$ axis, i.e., perpendicular to the axial image. The $4 \mathrm{Pi}$-confocal restored image reveals more details of the object than the confocal counterpart as a result of the improved $3 \mathrm{D}$ resolution. The actin fiber in the center is substantially better resolved.

with the 4Pi-PSF. The $X Z$ images are actually vertical cuts through the fibroblast $F$-actin skeleton. The $F$-actin fibers are perpendicular to the image plane, which is pointing in the $Y$ direction. Note the precision with which the single fiber in the center is localized and resolved in the 4Pi case. Again, this image should be contrasted with the two-photon confocal image which features a considerably stronger blur.

We evaluated the reduction in FWHM and found the lateral FWHM reduced by about 1.5. The FWHM along the optic axis was reduced six to sevenfold. The lateral and axial FWHM of the fibers in the image was 180 and $100 \mathrm{~nm}$, respectively. The $X Z$ images are actually part of 3D-data stacks consisting of $12 \mathrm{XZ}$ slices with $40 \mathrm{~nm}$ distance in $Y$ direction. This allowed us to display the actual threedimensional shape of the actin filaments in the imaged region. Figure 7 displays the 3D rendered actin for the (a) two-photon confocal and the (b) two-photon 4Pi-restored 
(a)

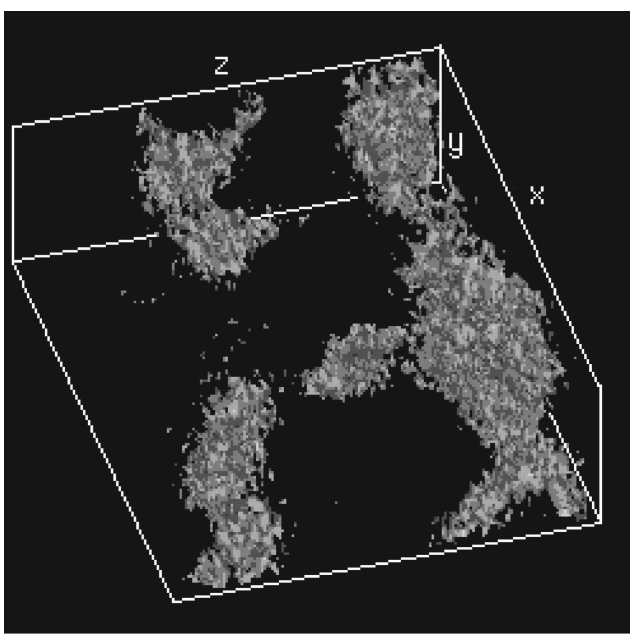

(b)

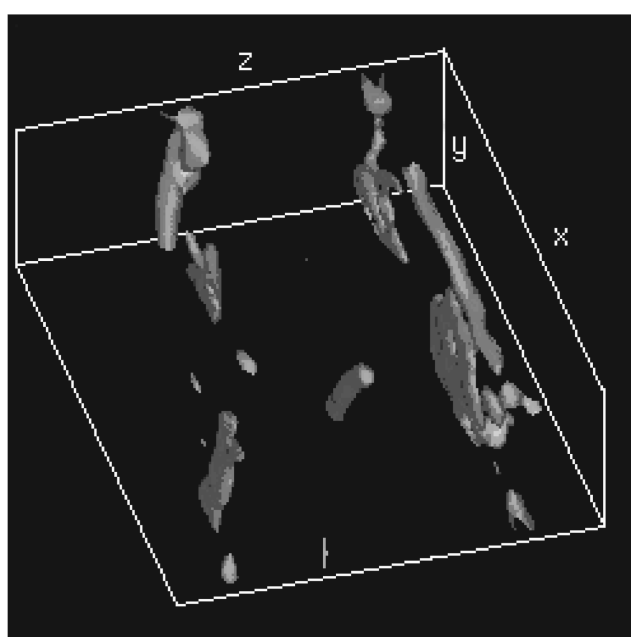

FIG. 7. Volume rendered 3D images of a (a) confocal, and (b) the correspondent 4Pi-confocal restored data stack. Both images were recorded with the same average power and the same pixel dwell time. The superior signalto-noise ratio in (b) is partly due to the increased signal in 4Pi-confocal imaging and partly due to the restoration procedure correcting for noise. The reduced uncertainty volume in (b) leads to a fundamentally improved imaging of the actin fibers in the cell volume. Note the vertical fiber in the center of the image.

case. Note the actin fiber in the center pointing (upwards) in $Y$ direction, of which Fig. 6 shows a section.

\section{DISCUSSION}

The improved resolution of the 4Pi-confocal microscope enables enhanced reproduction of the 3D structure of the object. It is interesting to note that in the 4Pi-confocal microscope, the axial resolution is better than its lateral counterpart. This is not surprising as it is a direct consequence of the improved aperture in the axial direction. Image restoration in 4Pi-confocal microscopy is very useful and efficient as it removes the lobes almost completely and provides superior resolution, in both axial and lateral direction.

We would like to emphasize that the resolution increase reported here cannot be attributed to the nonlinear nature of two-photon excitation, unless one expresses the achieved resolution in terms of a fraction of the wavelength. Usually, and particularly in our case, two-photon excitation does not lead to a sharper PSF in absolute values. ${ }^{17,44}$ This is because two-photon excitation requires a doubled excitation wavelength which results in a broader PSF. The role of twophoton excitation in our arrangement is to reduce the height of the side lobes of the 4Pi-PSF and to move the axial lobes further away from the focal plane. Thus the usefulness of confocal detection is as an efficient tool to suppress the lobes. ${ }^{32}$ We also note that the pulse duration of 250 fs of the excitation pulse is not critical since efficient two-photon microscopy of cultured cells can also be performed with much longer (picosecond) pulses. ${ }^{45}$

As pointed out in the introduction, the application of restoration procedures to standard confocal imaging also results in improved resolution. We also restored standard twophoton confocal images and found that the resolution improvement in that case is still remarkable, but less pronounced. We found an improvement by a factor of 1.5-2 in the $X$ and $Y$ directions, and 2 to 3 in its $Z$ counterpart. In 4Pi-confocal microscopes, the improvement is greater. From the PSF viewpoint, the 4Pi-PSF is sharper than the confocal PSF due to the 4.5 times narrower main maximum and also because of the lobe suppression. The sharp and spatially well-defined PSF increases the efficiency of the iterative approach of the restoration. From the viewpoint of the optical transfer function (OTF), the 4Pi-confocal microscope features a fourfold axial bandwidth in the axial direction, thus allowing for a considerably better axial restoration. We point out that with single-photon excitation and nonconfocal detection the OTF would have missing bands along the optic axis. This is because a nonconfocal single-photon excitation microscope does not feature axial discrimination by intensity. The role of two-photon excitation and confocal detection is to fill the missing bands in the 3D-OTF. This measure increases the efficiency of 3D-image restoration.

A further reason for the improved resolution is the high imaging fidelity as provided by stage scanning. ${ }^{19}$ Stage scanning allows for a high correction of the wave fronts and the involved optical components. In addition, the recording time for a single $X Z$ layer was $30 \mathrm{~s}$ which is about $30-50$ times longer than in beam scanning confocal microscopy. The recording times are about five times shorter than the typical recording time of a near-field fluorescence optical microscope. As with near-field optics, such recording times are justified when morphology is the main concern in imaging. Hence we are able to obtain a good signal-to-noise ratio (SNR) which is also an important reason for the effective resolution reported herein. As we have pointed out earlier, the synergistic combination of high quality imaging, high SNR, and subsequent efficient restoration is able to significantly increase the effective resolution in the far field. ${ }^{25,26,37}$ The concept of 4Pi-confocal microscopy also allows a beam scanning approach where two synchronized beams are scanned across the specimen for higher imaging speed.

A limitation of a nonlinear restoration procedure is that it is object dependent. For point-like objects, the restoration algorithm converges faster than for more complicated fluorophore distributions ${ }^{37}$ and it is also somewhat more efficient. This can be observed when comparing the data of the bead restoration with those of the biological sample. In the biological sample, the FWHM is about $20 \%$ larger than in 
the bead sample. Another reason for this phenomenon could be the fact that the PSFs were retrieved from the bead object which was immersed in Aquatex with a refractive index of $n \approx 1.5$ close to that of the oil/glass immersion system ( $n$ $=1.5)$. The biological samples were mounted in glycerol which has an index of refraction $(n=1.46)$ and is slightly mismatched to that of the oil. This mismatch induces a slight spherical aberration which is not accounted for in the PSF, with which we restored the biological images. Thus one can expect the restoration of the bead images to be slightly more efficient than that of the cell actin fibers. To obtain the best possible results, the use of a matched immersion system, i.e., glycerol immersion lenses, would be advantageous. This would provide a remedy for the remaining spherical aberration.

The obtained resolution shows that the use of a glycerol mountant is acceptable. However, we stress that the combination of oil immersion lenses and watery specimens ( $n$ $=1.33$ ) is not. For watery specimens, water immersion lenses will be naturally the lenses of choice. Obviously, the best results are expected for a complete match of the refractive index of the immersion system with that of the sample. Our experiments with beads indicate that for a well-matched system, point-like labels in the cytoskeleton could be imaged with a spatial resolution in the sub- $100 \mathrm{~nm}$ range (see Fig. 4).

The investigation of dense cell nuclei requires different fluorescence labels, usually blue fluorophores such as DAPI and Hoechst, and most probably a different compensation of the relative phase change. If the refractive index changes dramatically, the PSF undergoes changes which might also affect the restoration procedure. This could restrict the size of images to spatial dimensions where the PSF is sufficiently spatially invariant. One can expect that smaller images of about 2 to $3 \mu \mathrm{m}$ in size will be sufficient for distinguishing labels that are $0.1-0.3 \mu \mathrm{m}$ apart, which are otherwise indiscernible with focused light.

The efficiency of the restoration depends on the SNR. The latter can be increased by using a longer pixel dwell time. This is usually also the case in near-field fluorescence optics. For both methods, the possible increase in dwell time is limited by the finite cycles of excitations that a fluorescent molecule can undergo before decomposition. When comparing the signal to noise ratio of the two-photon confocal with that of its two-photon 4Pi-confocal images (Figs. 6 and 7) one notices improved signal to noise ratio of the latter. This is due to the fact that the interference of the focused beams leads to an increased local intensity in the focus resulting in a 2 to 3 times higher signal in two-photon fluorescence imaging. 4Pi-confocal microscopy also has another advantage in this respect. As the signal can be collected through both lenses one can further improve the signal height. 4Piconfocal microscopy thus improves both the resolution and the signal in far-field fluorescence microscopy.

\section{CONCLUSION}

Two-photon excitation 4Pi-confocal microscopy in combination with efficient image restoration has the potential to resolve fluorescent bead objects with a 3D resolution of 100 $\mathrm{nm}$ in three dimensions. The method is a genuine far-field approach and therefore applicable to imaging of the interior of three-dimensional structures such as cells. A useful criterion for three-dimensional resolution is the volume of uncertainty that can be calculated from the FWHM of the effective PSF of a far-field microscope. A direct comparison of the standard confocal and 4Pi-confocal restored results in a reduction of the uncertainty volume by more than one order of magnitude. This is, to our knowledge, the highest resolution in far-field light microscopy that has been achieved to date. These findings support our view that advanced far-field approaches are capable of setting new low benchmarks in light microscopy resolution and therefore complementing their near-field counterparts in high resolution applications.

${ }^{1}$ E. Abbe, Gesammelte Abhandlungen (Fischer, Jena, 1904).

${ }^{2}$ D. W. Pohl, W. Denk, and M. Lanz, Appl. Phys. Lett. 44, 651 (1984).

${ }^{3}$ A. Lewis, M. Isaacson, A. Harootunian, and A. Murray, Ultramicroscopy 13, 227 (1984).

${ }^{4}$ M. A. Paesler and P. J. Moyer, Near Field Optics Theory, Instrumentation, and Applications (Wiley, New York, 1996).

${ }^{5}$ J. Koglin, U. C. Fischer, and H. Fuchs, Phys. Rev. B 55, 7977 (1997).

${ }^{6}$ V. Sandoghar, S. Wegscheider, G. Krausch, and J. Mlynek, J. Appl. Phys. 81, 2499 (1997).

${ }^{7}$ J. Darnell, H. Lodish, and D. Baltimore, Molecular Biology of the Cell, 2nd ed. (Freeman, New York, 1990).

${ }^{8}$ J. Pawley, Handbook of Biological Confocal Microscopy (Plenum, New York, 1995).

${ }^{9}$ M. Minsky, in US Patent No. US3013467 1961.

${ }^{10} \mathrm{~T}$. Wilson and C. J. R. Sheppard, Theory and Practice of Scanning Optical Microscopy (Academic, New York, 1984).

${ }^{11}$ G. J. Brakenhoff, H. T. M. van der Voort, E. A. van Spronsen, W. A. M. Linnemans, and N. Nanninga, Nature (London) 317, 748 (1985).

${ }^{12}$ C. J. R. Sheppard and R. Kompfner, Appl. Opt. 17, 2879 (1978).

${ }^{13}$ W. Denk, J. H. Strickler, and W. W. Webb, Science 248, 73 (1990).

${ }^{14}$ S. W. Hell, K. Bahlmann, M. Schrader, A. Soini, H. Malak, I. Gryczynski, and J. R. Lakowicz, J. Biomed. Opt. 1, 71 (1996).

${ }^{15}$ D. L. Wokosin, V. E. Centonze, S. Crittenden, and J. White, Bioimaging 4, 208 (1996).

${ }^{16}$ M. Schrader, K. Bahlmann, and S. W. Hell, Optik (Stuttgart) 104, 116 (1997).

${ }^{17}$ C. J. R. Sheppard, Bioimaging 4, 124 (1996).

${ }^{18}$ S. W. Hell, in Europ. Patent No. EP0491289 1990.

${ }^{19}$ S. Hell and E. H. K. Stelzer, J. Opt. Soc. Am. A 9, 2159 (1992).

${ }^{20} \mathrm{M}$. Born and E. Wolf, Principles of Optics, 6th ed. (Pergamon, Oxford, 1993).

${ }^{21}$ T. Wilson, Confocal Microscopy (Academic, London, 1990).

${ }^{22}$ B. Bailey, D. L. Farkas, D. L. Taylor, and F. Lanni, Nature (London) 366, 44 (1993).

${ }^{23}$ E. H. K. Stelzer and S. Lindek, Opt. Commun. 111, 536 (1994).

${ }^{24}$ C. J. R. Sheppard, Opt. Commun. 119, 693 (1995).

${ }^{25}$ M. Schrader, S. W. Hell, and H. T. M. Van der Voort, Appl. Phys. Lett. 69, 3644 (1996).

${ }^{26}$ S. W. Hell, M. Schrader, and H. T. M. van der Voort, J. Microsc. 185, 1-5 (1997).

${ }^{27}$ K. R. Castleman, Digital Image Processing (Prentice-Hall, Englewood Cliffs, NJ, 1979).

${ }^{28}$ H. T. M. Van der Voort and K. C. Strasters, J. Microsc. 178, 165 (1995).

${ }^{29}$ S. W. Hell, M. Schrader, M. Nagorni, and H. T. M. van der Voort, Cell Vision -J. Anal. Morphol. 4, 187 (1997).

${ }^{30}$ B. Richards and E. Wolf, Proc. R. Soc. London, Ser. A 253, 358 (1959).

${ }^{31}$ S. W. Hell, S. Lindek, C. Cremer, and E. H. K. Stelzer, Appl. Phys. Lett. 64, 1335 (1994)

${ }^{32}$ S. Hell and E. H. K. Stelzer, Opt. Commun. 93, 277 (1992).

${ }^{33}$ S. W. Hell, S. Lindek, and E. H. K. Stelzer, J. Mod. Opt. 41, 675 (1994).

${ }^{34}$ P. E. Hänninen, S. W. Hell, J. Salo, E. Soini, and C. Cremer, Appl. Phys. Lett. 66, 1698 (1995). 
${ }^{35}$ D. A. Agard and J. W. Sedat, Nature (London) 302, 676 (1983).

${ }^{36}$ W. A. Carrington, R. M. Lynch, E. D. W. Moore, G. Isenberg, K. E. Fogarty, and F. S. Fay, Science 268, 1483 (1995).

${ }^{37}$ H. Kano, H. T. M. van der Voort, M. Schrader, G. M. P. van Kempen, and S. W. Hell, Bioimaging 4, 187 (1996).

${ }^{38}$ G. M. P. Van Kempen, H. T. M. van der Voort, J. G. J. Bauman, and K. C. Strasters, IEEE Eng. Med. Biol. Mag. 15, 76 (1996).

${ }^{39}$ I. Csiszar, Ann. Statistics 19, 2032 (1991).
${ }^{40}$ D. L. Snyder, T. J. Schutz, and J. A. O'Sullivan, IEEE Trans. Signal Process. 40, 1143 (1992).

${ }^{41}$ D. L. Snyder, A. M. Hammoud, and R. L. White, J. Opt. Soc. Am. A 10, 1014 (1993).

${ }^{42}$ M. Gu and C. J. R. Sheppard, J. Opt. Soc. Am. A 11, 1619 (1994).

${ }^{43}$ P. E. Hänninen and S. W. Hell, Bioimaging 2, 117 (1994).

${ }^{44}$ M. Gu and C. J. R. Sheppard, J. Microsc. 177, 128 (1995).

${ }^{45}$ J. Bewersdorf and S. W. Hell, J. Microsc. 191, 28 (1998). 